Modern Physics Letters A

(C) World Scientific Publishing Company

\title{
SELF-DUAL VORTICES IN THE ABELIAN CHERN-SIMONS MODEL WITH TWO COMPLEX SCALAR FIELDS
}

\author{
Yi-Shi Duan, Li-Da Zhang*'Yu-Xiao Liu \\ Institute of Theoretical Physics, Lanzhou University, \\ Lanzhou 730000, P. R. China
}

\begin{abstract}
Making use of $\phi$-mapping topological current method, we discuss the self-dual vortices in the Abelian Chern-Simons model with two complex scalar fields. For each scalar field, an exact nontrivial equation with a topological term which is missing in many references is derived analytically. The general angular momentum is obtained. The magnetic flux which relates the two scalar fields is calculated. Furthermore, we investigate the vortex evolution processes, and find that because of the present of the vortex molecule, these evolution processes is more complicated than the vortex evolution processes in the corresponding single scalar field model.
\end{abstract}

Keywords: Self-dual Vortices; Abelian Chern-Simons Model.

PACS numbers: 11.15.-q, 02.40.Pc, 47.32.Cc

\section{INTRODUCTION}

The (2+1)-dimensional physics system presents many interesting surprises, both experimentally and theoretically. For example, in last three decades, it has been realized that while in three and higher space dimensions the particles must be either bosons or fermions, in two space dimensions the particles can have any fractional spin and can satisfy any fractional statistics which interpolates between the familiar two 1 The particles obeying such statistics are called as anyons, whose existence in real physics system have been proved by the fractional quantum Hall effect ${ }^{2 \mid 3}$ where anyons act as fractionally charged excitations of the imcompressible quantum fluid.

In (2+1)-dimensional spacetime, a new type of gauge theory named ChernSimons theory which is different from Maxwell theory plays a very significant role in many aspects. 14 One of the most remarkable properties of the Chern-Simons action is that it does not depends on the metric tensor, and always has the same form no matter the spacetime is flat or not. Hence, the Chern-Simons theory is an

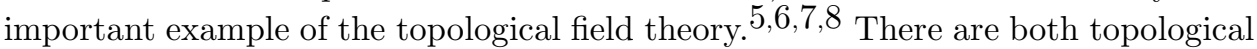
and nontopological self-dual vortex solutions in the Chern-Simons model $\frac{910}{}$ And topology is indispensable even in the situation where only the nontopological vortex solutions are present.

${ }^{*}$ Corresponding author. Email: zhangld04@lzu.cn 
On the other hand, for over four decades there has been a wide interest in the condensed-matter systems with several coexisting Bose condensates. ${ }^{11}$ Recent research 12 has revealed that a charged two-condensate Ginzburg-Landau model can be mapped onto a version of the nonlinear $O(3) \sigma$ model. This leads to many efforts to study the topological property behind the two-condensate system. $13|14| 15 \mid 16$

In light of $\phi$-mapping topological current method, 17 the present paper investigates the self-dual vortices in the Abelian Chern-Simons model with two complex scalar fields, which is a kind of $(2+1)$-dimensional field theory generalization of the two-condensate system. To be self-contained basically, in Sec. 2 we briefly review the $\phi$-mapping topological current method. In Sec. 3 we introduce the model to be considered, and derive a set of self-dual equations. In Sec. 4. we find a nontrivial equation with a topological term for each scalar field, which is the development of our previous work, $\frac{18 \mid 1920}{20}$ and also obtain an equation which relates the two scalar fields by their topological terms. Besides, the angular momentum and the magnetic flux of the system are calculated. In Sec. 5, we briefly consider the evolution processes of the vortices, and find that because of the present of the vortex molecule, the detailed evolution processes of the vortices in the double scalar field model is more complicated than the vortex evolution processes in the corresponding single scalar field model, which have been investigated in Ref. 21 in detail.

\section{A BRIEF REVIEW OF $\phi$-MAPPING TOPOLOGICAL CURRENT METHOD}

Because the $\phi$-mapping topological current method is unfamiliar to most of the readers, we will briefly review it in the following. Consider a D-dimensional smooth manifold with metric tensor $g_{\mu \nu}$ and local coordinates $x^{\mu}$. Define a map $\Phi$ :

$$
\Phi^{a}=\Phi^{a}\left(x^{\mu}\right) \quad(a=1, \cdots, d<D),
$$

and introduce the direction unit field of $\Phi^{a}$ :

$$
N^{a}=\frac{\Phi^{a}}{\|\Phi\|} \quad\left(\|\Phi\|=\sqrt{\Phi^{a} \Phi^{a}}\right) .
$$

Using $N^{a}$, we can construct a topological tensor current:

$$
j^{\mu \cdots \nu} \sim \frac{1}{\sqrt{\operatorname{detg}_{\mu \nu}}} \epsilon^{\mu \cdots \nu \lambda \cdots \rho} \epsilon^{a \cdots b} \partial_{\lambda} N^{a} \cdots \partial_{\rho} N^{b} .
$$

It is not difficult to find that $j^{\mu \cdots \nu}$ is completely antisymmetric and identically conserved. Defined the Jacobian tensor $D^{\mu \cdots \nu}\left(\frac{\Phi}{x}\right)=\frac{1}{d !} \epsilon^{\mu \cdots \nu \lambda \cdots \rho} \epsilon^{a \cdots b} \partial_{\lambda} \Phi^{a} \cdots \partial_{\rho} \Phi^{b}$, and the $\phi$-space Green function $G_{d}(\|\Phi\|)=\left\{\begin{array}{l}\frac{1}{\|\Phi\|^{d-2}}, d>2 ; \\ \ln \|\Phi\|, d=2 .\end{array}\right.$ Then using $\partial_{\mu} N^{a}=$ $\frac{1}{\|\Phi\|} \partial_{\mu} \Phi^{a}+\Phi^{a} \partial_{\mu} \frac{1}{\|\Phi\|}$ and the Green function relation $\frac{\partial}{\partial \Phi^{a}} \frac{\partial}{\partial \Phi^{a}} G_{d}(\|\Phi\|) \sim \delta^{d}(\Phi)$ we can find

$$
j^{\mu \cdots \nu} \sim \frac{1}{\sqrt{\operatorname{detg}_{\mu \nu}}} \delta^{d}(\Phi) D^{\mu \cdots \nu}\left(\frac{\Phi}{x}\right) .
$$


The $\delta$-function in Eq. (4) implies that $j^{\mu \cdots \nu} \neq 0$ only at the points where $\Phi^{a}=0$. Generally, these points would correspond to the submanifolds where the topological defects are located. Denoting the $k$-th above-mentioned submanifold by $M_{k}$, and defining a corresponding normal submanifold $N_{k}$ which is spanned by the parameter $v^{A}(A=1, \cdots, k)$ with the metric tensor $g_{A B}$, we can get the only intersection point of $M_{k}$ and $N_{k}$ denoted by $p_{k}$. According to the implicit function theorem, 22 at the regular points of $\Phi^{a}$, there exists the only solution to the equations $\Phi^{a}=0$, and Eq. (41) can be expanded as

$$
j^{\mu \cdots \nu} \sim \frac{1}{\sqrt{\operatorname{det} g_{\mu \nu}}} \sum_{k} \frac{W_{k} \sqrt{\operatorname{detg}_{A B}}}{\left.D\left(\frac{\Phi}{v}\right)\right|_{p_{k}}} \delta^{d}\left(M_{k}\right) D^{\mu \cdots \nu}\left(\frac{\Phi}{x}\right),
$$

where the Jacobian $D\left(\frac{\Phi}{v}\right)=\frac{1}{d !} \epsilon^{A \cdots B} \epsilon^{a \cdots b} \partial_{A} \Phi^{a} \cdots \partial_{B} \Phi^{b}, \delta^{d}\left(M_{k}\right)$ is the $\delta$-function on the submanifold $M_{k}$, and $W_{k}$ denotes the winding number of the $k$-th topological defect. Some important physical quantities concerning the topology, say magnetic flux, can be calculated from Eq. (5). By virtue of the implicit function theorem, 22 the irregular points of $\Phi^{a}$ correspond to the branch points of the topological current $j^{\mu \cdots \nu}$, and the branch processes of $j^{\mu \cdots \nu}$ occur at these very points. These branch processes can describe various evolutions of the topological defects, and the total topological charge of the system will keep unchange during these evolutions since $j^{\mu \cdots \nu}$ is a conserved current.

\section{THE MODEL}

We consider the $(2+1)$-dimensional Chern-Simons system described by the Lagrangian

$$
\mathcal{L}=\frac{1}{2} \kappa \varepsilon^{\mu \nu \rho} a_{\mu} \partial_{\nu} a_{\rho}+\left|D_{\mu} \psi\right|^{2}+\left|D_{\mu} \phi\right|^{2}-U(\psi, \phi),
$$

where $D_{\mu} \psi=\left(\partial_{\mu}-i e_{1} a_{\mu}\right) \psi, D_{\mu} \phi=\left(\partial_{\mu}-i e_{2} a_{\mu}\right) \phi$, and

$$
U(\psi, \phi)=\frac{1}{\kappa^{2}}\left(e_{1}^{2}|\psi|^{2}+e_{2}^{2}|\phi|^{2}\right)\left(e_{1}|\psi|^{2}+e_{2}|\phi|^{2}-v^{2}\right)^{2} .
$$

Here, coupling $e_{1}, e_{2}>0, \psi$ and $\phi$ are complex scalar fields, $a_{\mu}$ denotes the ChernSimons gauge field. The specific form of the potential (7) gives rise to a self-dual system.

The variation of the action with respect to $a_{0}$ leads to a Gauss law:

$$
\kappa f_{12}=e_{1} J_{\psi}^{0}+e_{2} J_{\phi}^{0},
$$

where $f_{\mu \nu}=\partial_{\mu} a_{\nu}-\partial_{\nu} a_{\mu}, J_{\psi}^{\mu}=-i\left[\psi^{*} D^{\mu} \psi-\left(D^{\mu} \psi\right)^{*} \psi\right]$ and $J_{\phi}^{\mu}=-i\left[\phi^{*} D^{\mu} \phi-\right.$ $\left.\left(D^{\mu} \phi\right)^{*} \phi\right]$. For finite-energy configurations, the magnetic flux $\Phi=\int d^{2} x f_{12}$ and charges $Q_{\psi}=\int d^{2} x J_{\psi}^{0}$ and $Q_{\phi}=\int d^{2} x J_{\phi}^{0}$ are related by Eq. (8), namely

$$
\kappa \Phi=e_{1} Q_{\psi}+e_{2} Q_{\phi} .
$$


Set $i, j=1,2$, the energy function is

$$
E=\int d^{2} x\left[\left|D_{0} \psi\right|^{2}+\left|D_{0} \phi\right|^{2}+\left|D_{i} \psi\right|^{2}+\left|D_{i} \phi\right|^{2}+U\right] .
$$

Employing the Gauss laws and integrating by parts, one obtains the bound

$$
E \geq v^{2}|\Phi| \text {. }
$$

This bound is saturated by the fields satisfying the following self-dual equations:

$$
\begin{aligned}
& \left(D_{1} \pm i D_{2}\right) \psi=\left(D_{1} \pm i D_{2}\right) \phi=0, \\
& D_{0} \psi \pm i \frac{e_{1}}{\kappa} \psi\left(e_{1}|\psi|^{2}+e_{2}|\phi|^{2}-v^{2}\right)=0, \\
& D_{0} \phi \pm i \frac{e_{2}}{\kappa} \phi\left(e_{1}|\psi|^{2}+e_{2}|\phi|^{2}-v^{2}\right)=0 .
\end{aligned}
$$

\section{STATIC VORTICES}

From the potential (7), it is obvious that there is a symmetric phase as well as an asymmetric or broken phase. In the asymmetric phase, static finite energy solutions require both scalar fields $\psi$ and $\phi$ to be at most a pure phase on the circle at infinity, i.e. the vacuum manifold for both fields $\psi$ and $\phi$ is $U(1) \simeq S^{1}$. So the first homotopy group $\pi_{1}(U(1))=Z$ provides the topological guarantee for the existence of the vortex solutions. In the symmetric phase, nontopological static soliton solutions are possible, which make the situation more complicated than the self-dual Maxwell system.

For static case, from Eq. (8) we obtain

$$
a_{0}=-\frac{\kappa f_{12}}{2\left(e_{1}^{2}|\psi|^{2}+e_{2}^{2}|\phi|^{2}\right)} .
$$

From Eq. (12), we have

$$
e_{1} a_{i}= \pm \epsilon^{0 i j} \partial_{j} \ln |\psi|-\frac{i}{2}\left(\frac{\psi^{*}}{|\psi|} \partial_{i} \frac{\psi}{|\psi|}-\frac{\psi}{|\psi|} \partial_{i} \frac{\psi^{*}}{|\psi|}\right)
$$

and furthermore

$$
e_{1} f_{12}=e_{1} \epsilon^{0 i j} \partial_{i} a_{j}=\mp \partial_{i} \partial_{i} \ln |\psi|-i \epsilon^{0 i j} \partial_{i} \frac{\psi^{*}}{|\psi|} \partial_{j} \frac{\psi}{|\psi|} .
$$

At this point, expressing $\psi$ as $\psi=\psi^{1}+i \psi^{2}$, we can write the second term on the right-hand side of Eq. (15) as $\epsilon^{0 i j} \epsilon_{a b} \partial_{i}\left(\psi^{a} /|\psi|\right) \partial_{j}\left(\psi^{b} /|\psi|\right)$. This expression has an exact form of topological current (3). So, using the $\phi$-mapping method, we can rewrite Eq. (15) as

$$
e_{1} f_{12}=e_{1} \epsilon^{0 i j} \partial_{i} a_{j}=\mp \nabla^{2} \ln |\psi|+2 \pi \delta(\psi) D\left(\frac{\psi}{x}\right),
$$

where the Jacobian $D\left(\frac{\psi}{x}\right)=\frac{1}{2} \epsilon^{0 i j} \epsilon_{a b} \partial_{i} \psi^{a} \partial_{j} \psi^{b}$ and $\delta(\psi)$ is the $\delta$-function in $\psi$ space. The presence of $\delta(\psi)$ in Eq. (16) identifies the existence of the vortex solutions in model (6), and implies that the cores of the vortices locate at the zeros of $\psi$. To 
be specific, we denote the isolated zeros of $\psi$ by $\vec{z}_{r}(r=1, \ldots, m)$ and the isolated zeros of $\phi$ by $\vec{z}_{s}(s=1, \ldots, n)$. According to the implicit function theorem, 22 when the zeros $\vec{z}_{r}$ are the regular points of $\psi$, i.e. when

$$
\left.D\left(\frac{\psi}{x}\right)\right|_{\vec{z}_{r}} \neq 0
$$

there exists one and only one continuous vortex solution to the equations $\psi^{a}=$ $0(a=1,2)$. Furthermore, under the condition (17), we can express $\delta(\psi)$ as $23 \mid 24$

$$
\delta(\psi)=\sum_{r=1}^{m} \frac{W_{r}}{\left.D\left(\frac{\psi}{x}\right)\right|_{\vec{z}_{r}}} \delta\left(\overrightarrow{x_{r}}-\vec{z}_{r}\right)
$$

where $W_{r}$ is the winding number of the $r$-th isolated zero of $\psi$. If we consider the case with $\psi$ vanishing at infinity in the following way

$$
|\psi| \rightarrow \frac{1}{r^{\alpha}} \quad \text { as } r \rightarrow \infty,
$$

where $\alpha>0$. Then

$$
\delta(\psi) D\left(\frac{\psi}{x}\right) \rightarrow 0 \quad \text { as } r \rightarrow \infty .
$$

Therefore Eq. (16) can generally be rewritten as

$$
e_{1} f_{12}=\mp \nabla^{2} \ln |\psi|+2 \pi \sum_{r=1}^{m} W_{r} \delta\left(\vec{x}-\vec{z}_{r}\right)
$$

even though $\psi$ vanishes at infinity in some kind of specific way.

Using Eqs. (12), (13) and (21), we find a nontrivial equation to be satisfied by $\psi$ :

$$
\nabla^{2} \ln |\psi|^{2}-\frac{4 e_{1}}{\kappa^{2}}\left(e_{1}^{2}|\psi|^{2}+e_{2}^{2}|\phi|^{2}\right)\left(e_{1}|\psi|^{2}+e_{2}|\phi|^{2}-v^{2}\right)= \pm 4 \pi \sum_{r=1}^{m} W_{r} \delta\left(\vec{x}-\vec{z}_{r}\right) .
$$

The above nontrivial equation includes a topological term on its right-hand side, which is missing in many references. Under $e_{1} \leftrightarrow e_{2}, \psi \leftrightarrow \phi, \vec{z}_{r} \leftrightarrow \overrightarrow{z_{s}}$ and $W_{r} \leftrightarrow W_{s}$ (the winding number of the $s$-th isolated zero of $\phi$ ), all conclusions and expressions we obtain above still hold for $\phi$. Especially, corresponding to Eq. (22), we have

$$
\nabla^{2} \ln |\phi|^{2}-\frac{4 e_{2}}{\kappa^{2}}\left(e_{1}^{2}|\psi|^{2}+e_{2}^{2}|\phi|^{2}\right)\left(e_{1}|\psi|^{2}+e_{2}|\phi|^{2}-v^{2}\right)= \pm 4 \pi \sum_{s=1}^{n} W_{s} \delta\left(\vec{x}-\overrightarrow{z_{s}}\right) .
$$

From Eqs. (22) and (23), we find the following equation for $\psi$ and $\phi$ :

$$
\nabla^{2} \ln \frac{|\psi|^{e_{2}}}{|\phi|^{e_{1}}}= \pm 2 \pi\left[e_{2} \sum_{r=1}^{m} W_{r} \delta\left(\vec{x}-\vec{z}_{r}\right)-e_{1} \sum_{s=1}^{n} W_{s} \delta\left(\vec{x}-\overrightarrow{z_{s}}\right)\right] .
$$

Obviously, the above equation relates $\psi$ and $\phi$ by their topological terms, and displays the topological relation between them. 
A characteristic feature of Chern-Simons vortices is that they carry nonzero angular momentum which is given by

$$
J=-\int d^{2} x \epsilon^{i j} x^{i}\left[\left(D_{0} \psi\right)^{*} D_{j} \psi+\left(D_{0} \phi\right)^{*} D_{j} \phi+\text { c.c. }\right] .
$$

Furthermore, from Eq. (14) and its $\phi$ counterpart as well as Eq. (12), Eq. (25) becomes

$$
\begin{aligned}
J & =-2 \int d^{2} x \epsilon^{i j} x^{i} a_{0}\left[|\psi|^{2}\left(e_{1}^{2} a_{j}-e_{1} \epsilon^{a b} \hat{\psi}^{a} \partial_{j} \hat{\psi}^{b}\right)+|\phi|^{2}\left(e_{2}^{2} a_{j}-e_{2} \epsilon^{a b} \hat{\phi}^{a} \partial_{j} \hat{\phi}^{b}\right)\right] \\
& =\frac{1}{\kappa} \int d^{2} x x^{i}\left(e_{1}|\psi|^{2}+e_{2}|\phi|^{2}-v^{2}\right) \partial_{i}\left(e_{1}|\psi|^{2}+e_{2}|\phi|^{2}\right) \\
& = \begin{cases}-\frac{1}{\kappa} \int d^{2} x\left(e_{1}|\psi|^{2}+e_{2}|\phi|^{2}-v^{2}\right)^{2}, & \text { asymmetric phase; } \\
-\frac{1}{\kappa} \int d^{2} x\left(e_{1}|\psi|^{2}+e_{2}|\phi|^{2}\right)\left(e_{1}|\psi|^{2}+e_{2}|\phi|^{2}-2 v^{2}\right), & \text { symmetric phase. }\end{cases}
\end{aligned}
$$

This conclusion is exactly the generalization of the angular momentum of single complex scalar Chern-Simons vortices. 25

In the following, we concentrate on asymmetric phase and assume

$$
\left(\begin{array}{c}
\psi \\
\phi
\end{array}\right) \rightarrow\left(\begin{array}{c}
\psi_{0} \\
\phi_{0}
\end{array}\right) \quad \text { as } r \rightarrow \infty
$$

where $\psi_{0}$ and $\phi_{0}$ satisfy $e_{1}\left|\psi_{0}\right|^{2}+e_{2}\left|\phi_{0}\right|^{2}=u^{2}$. Because different choices for the vacuum values are inequivalent, we need consider the case with $\psi_{0} \phi_{0} \neq 0$ and the case with one of the vacuum values is zero respectively.

In the case with $\psi_{0} \phi_{0} \neq 0$, from Eq. (21) and its $\phi$ counterpart, the flux is given by

$$
\Phi=\int d^{2} x f_{12}=\frac{2 \pi \sum_{r=1}^{m} W_{r}}{e_{1}}=\frac{2 \pi \sum_{s=1}^{n} W_{s}^{\prime}}{e_{2}},
$$

which implies that the regular vortex solutions may exist only if $e_{1} / e_{2}$ is rational and equals to the ratio of the total winding number of zeros of $\psi$ and $\phi$. On the other hand, in the case with one of the scalar fields, say $\psi$, vanishes at infinity in the way of Eq. (19), from Eq. (21) and its $\phi$ counterpart the flux will be

$$
\Phi=\frac{2 \pi\left(\sum_{r=1}^{m} W_{r} \pm \alpha\right)}{e_{1}}=\frac{2 \pi \sum_{s=1}^{n} W_{s}}{e_{2}},
$$

where $+\alpha(-\alpha)$ is for $\sum_{r=1}^{m} W_{r}>0(<0)$.

For the nontopological soliton solutions allowed in symmetric phase, the situation is different from the topological soliton solutions in some aspects. For example, suppose that for $\alpha>0$ and $\beta>0$

$$
\begin{gathered}
|\psi| \rightarrow \frac{1}{r^{\alpha}} \quad \text { as } r \rightarrow \infty, \\
|\phi| \rightarrow \frac{1}{r^{\beta}} \quad \text { as } r \rightarrow \infty .
\end{gathered}
$$


Then, as before, we can find the flux is given by

$$
\Phi=\frac{2 \pi\left(\sum_{r=1}^{m} W_{r} \pm \alpha\right)}{e_{1}}=\frac{2 \pi\left(\sum_{s=1}^{n} W_{s} \pm \beta\right)}{e_{2}},
$$

where $+\alpha(-\alpha)$ is for $\sum_{r=1}^{m} W_{r}>0(<0)$ and $+\beta(-\beta)$ is for $\sum_{s=1}^{n} W_{s}>0(<0)$.

\section{EVOLUTION PROCESSES OF VORTICES}

In Ref. 21, the evolution of the Chern-Simons vortices in the model with single complex scalar field was discussed in detail. As for the present system, we can directly generalize the results in Ref. 21 to the vortex evolution processes concerning the individual scalar field, say $\psi$. The key issue here is the evolution processes concerning both $\psi$ and $\phi$.

For complex scalar field $\psi$, define topological current

$$
j^{\mu}=\frac{1}{2 \pi} \epsilon^{\mu \nu \lambda} \epsilon_{a b} \partial_{\nu} \frac{\psi^{a}}{|\psi|} \partial_{\lambda} \frac{\psi^{b}}{|\psi|} .
$$

It is easy to see that $j^{\mu}$ is identically conserved, i.e. $\partial_{\mu} j^{\mu}=0$. Similar to the case in Sec. 4. we can obtain $j^{\mu}=\delta(\psi) D^{\mu}\left(\frac{\psi}{x}\right)$. According to the implicit function theorem as before, when

$$
\left.D\left(\frac{\psi}{x}\right)\right|_{\left(\vec{z}_{r}, t\right)} \neq 0
$$

there exists one and only one continuous vortex solution to the equations:

$$
\begin{aligned}
& \psi^{1}\left(x^{1}, x^{2}, t\right)=0, \\
& \psi^{2}\left(x^{1}, x^{2}, t\right)=0,
\end{aligned}
$$

which can be expressed as

$$
\vec{z}_{r}=\vec{z}_{r}(t)
$$

and $\delta(\psi)$ can be expressed as

$$
\delta(\psi)=\sum_{r=1}^{m} \frac{W_{r}}{\left.D\left(\frac{\psi}{x}\right)\right|_{\left(\vec{z}_{r}, t^{*}\right)}} \delta\left(\overrightarrow{x_{r}}-\vec{z}_{r}\right) .
$$

Using Eqs. (32) and (37), we can find the topological charge corresponding to the current $j^{\mu}$ is the winding number $W_{r}$. Since the topological current $j^{\mu}$ is identically conserved, the topological charge $W_{r}$ will remain unchanged in various evolution processes. Obviously, the above discussion also apply to $\phi$. Therefore, at this level, the topological evolution of the vortices for $\psi$ and $\phi$ is relatively independent.

At this point, a question rises: is there any other nontrivial topological configuration formed in the system due to the interaction between the fields $\psi$ and $\phi$ ? To answer this question, let us pay attention to coherently coupled two-component Bose-Einstein condensates studied in Ref. 26. Such a system is not described by a 
gauge theory, but it has the same complex scalar field structure with the present system. So we can use the method presented in Ref. 26 to get an insight into the topological configuration formed by the internal coherent coupling between $\psi$ and $\phi$. To be specific, writing $\psi=\rho \cos (\theta / 2) \exp \left(i \varphi_{1}\right)$ and $\phi=\rho \sin (\theta / 2) \exp \left(i \varphi_{2}\right)$, we can introduce an internal space vector

$$
\vec{n}=(\sin \theta \cos \gamma, \sin \theta \sin \gamma, \cos \theta),
$$

where $\gamma=\varphi_{2}-\varphi_{1}$. Field $\psi$ and $\phi$ tend to their vacuum values at the space infinity. This boundary condition compactifies space $R^{2}$ into $S^{2}$. So vector $\vec{n}$ defines a mapping: $S^{2} \rightarrow S^{2}$. Since $\pi_{2}\left(S^{2}\right)=Z$, there exists another kind of topological soliton named skyrmions. In the general nonaxisymmetric case, such a skyrmion will split into a pair of vortex-antivortex, or a vortex molecule, which is formed by a vortex in $\psi(\phi)$ and an antivortex in $\phi(\psi)$. The concrete amounts of the bound vortex molecules and unbound vortices in the system depend on the winding numbers of $\psi$ and $\phi$ as well as the skyrmion numbers of the system.

Because of the existence of the vortex molecule, the detailed evolution processes of the vortices in model (6) is more complicated than the vortex evolution processes in the corresponding single scalar field model. For example, the processes in which one vortex in a vortex molecule is replaced by another vortex is absent in the single scalar field case. We will leave this subject to the future studies.

\section{CONCLUSIONS}

Making use of $\phi$-mapping topological current method, we have discussed the selfdual vortices in the Abelian Chern-Simons model with the Chern-Simons gauge field coupling to two complex scalar fields. This model is a kind of (2+1)-dimensional field theory generalization of the two-condensate system, which receives wide attention in condensed-matter physics. $\frac{13 \sqrt{14}[15] 16}{16}$ For each scalar field, we analytically derived an exact nontrivial equation with a topological term which is missing in many references. Each of these equations holds for a very wide range of the boundary conditions. Besides, the equation relating the two scalar fields by their topological terms was also obtained. We calculated the angular momentum of the Chern-Simons vortices, and the result is exactly the generalization of the angular momentum of single scalar field Chern-Simons vortices. We also calculated the magnetic flux of the system for different boundary conditions, and found this magnetic flux relates the total winding numbers for the two scalar fields in a specific way which depends on the given boundary condition. Furthermore, we briefly discussed the evolution processes of the vortices, and found that because of the present of the vortex molecule, the detailed evolution processes of the vortices in the present model is more complicated than the vortex evolution processes in the corresponding single scalar field model. 


\section{Acknowledgements}

It is a pleasure to thank Dr. Yong-Qiang Wang for discussions. This work was supported by the National Natural Science Foundation of the People's Republic of China (No. 502-041016 and No. 10705013) and the Fundamental Research Fund for Physics and Mathematics of Lanzhou University (No. Lzu07002).

\section{References}

1. A. Khare, Fractional Statistics and Quantum Theory, (World Scientific, Beijing, 1997).

2. D. C. Tsui, H. L. Stormer and A. C. Gossard, Phys. Rev. Lett. 48 (1982) 1559.

3. R. B. Laughlin, Phys. Rev. Lett. 50 (1983) 1395.

4. G. V. Dunne, Aspects of Chern-Simons theory, hep-th/9902115.

5. A. S. Schwarz, Lett. Math. Phys. 2 (1978) 247.

6. E. Witten, Comm. Math. Phys. 121 (1989) 351.

7. M. Bos and V. P. Nair, Phys. Lett. B223 (1989) 61.

8. D. Birmingham, M. Blau, M. Rakowsky and G. Thompson, Phys. Rep. 209 (1991) 129.

9. J. Hong, Y. Kim and P. Y. Pac, Phys. Rev. Lett. 64 (1990) 2230.

10. R. Jackiw and E. J. Weinberg, Phys. Rev. Lett. 64 (1990) 2234.

11. H. Suhl, B.T. Matthias and L.R. Walker, Phys. Rev. Lett. 3 (1959) 552.

12. E. Babaev, L. D. Faddeev and A. J. Niemi, Phys. Rev. B65 (2002) 100512, cond-mat/0106152.

13. E. Babaev, Phys. Rev. Lett. 88 (2002) 177002, cond-mat/0106360.

14. E. Babaev, Phys. Rev. Lett. 89 (2002) 067001, cond-mat/0111192.

15. Y. Jiang, Phys. Rev. B70 (2004) 012501, cond-mat/0410121.

16. Y. S. Duan, X. H. Zhang, Y. X. Liu and L. Zhao, Phys. Rev. B74 (2006) 144508, cond-mat/0703475.

17. Y. S. Duan, L. B. Fu and G. Jia, J. Math. Phys. 41 (2000) 4379, hep-th/9904123.

18. Y. Q. Wang, Y. X. Liu and Y. S. Duan, A new form of self-duality equations with topological term, hep-th/0508104.

19. Y. Q. Wang, T. Y. Si, Y. X. Liu and Y. S. Duan, Mod.Phys.Lett. A20 (2005) 3045, hep-th/0508111.

20. Y. X. Liu, L. Zhao, X. H. Zhang and Y. S. Duan, Nucl. Phys. B785 (2007) 234, arXiv:0704.2812 [hep-th].

21. L. B. Fu, Y. S. Duan and H. Zhang, Phys. Rev. D61 (2000) 045004, hep-th/0112033.

22. É. Goursat, A Course in Mathematical Analysis, vol. I, translated by E. R. Hedrich, (Dover, New York, 1904).

23. J. A. Schouten, TensorAnalysis for Physicist, (Clarendon, Oxfort, 1951).

24. Y. S. Duan and X. Liu, JHEP 0402 (2004) 028, hep-th/0304146.

25. R. Jackiw, K. Lee and E. J. Weinberg, Phys. Rev. D42 (1990) 3488.

26. K. Kasamatsu, M. Tsubota and M. Ueda, Phys. Rev. Lett. 93 (2004) 250406, cond-mat/0406150. 